\title{
THE UNCONDITIONAL BASIC SEQUENCE PROBLEM
}

\author{
W. T. GOWERS AND B. MAUREY
}

\section{INTRODUCTION}

A fundamental role in the theory of Banach spaces is played by the notion of a Schauder basis. If $X$ is a Banach space then a sequence $\left(x_{n}\right)_{n=1}^{\infty}$ is a Schauder basis (or simply a basis) of $X$ if every $x \in X$ can be written in a unique way as a norm-convergent sum of the form $\sum_{n=1}^{\infty} a_{n} x_{n}$. This definition clearly depends very much on the order of the $x_{n}$, and it is certainly possible for a permutation of a basis to fail to be a basis. On the other hand, many bases that occur naturally, such as the standard basis of $\ell_{p}$ when $1 \leqslant p<\infty$, are bases under any permutation. It is therefore natural to give a name to this special kind of basis. As it happens there are several equivalent definitions.

Theorem 1. Let $X$ be a (real or complex) Banach space and let $\left(x_{n}\right)_{n=1}^{\infty}$ be a basis of $X$. Then the following are equivalent.

(i) $\left(x_{\pi(n)}\right)_{n=1}^{\infty}$ is a basis of $X$ for every permutation $\pi: \mathbb{N} \rightarrow \mathbb{N}$.

(ii) Sums of the form $\sum_{n=1}^{\infty} a_{n} x_{n}$ converge unconditionally whenever they converge.

(iii) There exists a constant $C$ such that, for every sequence of scalars $\left(a_{n}\right)_{n=1}^{\infty}$ and every sequence of scalars $\left(\epsilon_{n}\right)_{n=1}^{\infty}$ of modulus at most 1 , we have the inequality

$$
\left\|\sum_{n=1}^{\infty} \epsilon_{n} a_{n} x_{n}\right\| \leqslant C\left\|\sum_{n=1}^{\infty} a_{n} x_{n}\right\| .
$$

A basis satisfying these conditions is called an unconditional basis, and a basis satisfying the third condition for some given constant $C$ is called $C$ unconditional. An infinite sequence that is a basis of its closed linear span is called a basic sequence; if it is an unconditional basis of its closed linear span then it is an unconditional basic sequence. The basic facts about such sequences, including Theorem 1, can be found in [LT].

For a long time a major unsolved problem was whether every separable $\mathrm{Ba}$ nach space had a basis. This question was answered negatively by Enflo in 1973 [E]. On the other hand, it is not hard to show that every space contains a basic sequence. Spaces with unconditional bases have much more structure than general spaces, so examples of spaces without them are easy to find. Indeed, the spaces $C([0,1])$ and $L_{1}$ do not have an unconditional basis. This leaves the

Received by the editors September 3, 1992.

1991 Mathematics Subject Classification. Primary 46B15; Secondary 46B20,46B45. 
question of whether every space contains an unconditional basic sequence or, equivalently, has an infinite-dimensional subspace with an unconditional basis. The earliest reference we know for the problem is [BP] (1958), where it appears as problem 5.1 (although Mazur was aware of the question at least ten years earlier); actually we solve the more precise problem 5.11 , since our example is a reflexive Banach space. The easier related problem 5.12, whether every normalized weakly null sequence has an unconditional subsequence, was solved many years ago [MR].

In the summer of 1991, the first-named author found a counterexample. A short time afterward the second-named author independently found a counterexample as well. On comparing our examples, we discovered that they were almost identical, as were the proofs that they were indeed counterexamples, so we decided to publish jointly and work together on further properties of the space. As a result of our collaboration, the proofs of some of the main lemmas have been simplified and tightened.

After reading our original preprints, W. B. Johnson pointed out that our proof(s) could be modified to give a much stronger property of the space. Lindenstrauss had asked [L] whether every infinite-dimensional Banach space was decomposable, that is, could be written as a topological direct sum $Y \oplus Z$ with $Y$ and $Z$ infinite-dimensional. Johnson's observation was that our space, which for the remainder of the introduction we shall call $X$, is not only not decomposable but does not even have a decomposable subspace. That is, $X$ is hereditarily indecomposable or H.I. Equivalently, if $Y$ and $Z$ are two infinite-dimensional subspaces of $X$ and $\epsilon>0$ then there exist $y \in Y$ and $z \in Z$ such that $\|y\|=\|z\|=1$ and $\|y-z\|<\epsilon$. This turned out to be a key property of $X$ in that all of the pathological properties that we know about $X$ can be deduced from the fact that it is H.I. In particular, it is easy to see that a space with this property cannot contain an unconditional basic sequence.

Another immediate consequence is that either the space is a new prime $\mathrm{Ba}$ nach space (which means that it is isomorphic to all its complemented subspaces), or it fails to be isomorphic to a subspace of finite codimension. If the second statement is true then it must fail to be isomorphic to a subspace of codimension one, giving a counterexample to a question of Banach which has come to be known as the hyperplane problem. The first author modified the construction of $X$ to give such a counterexample, and, in fact, one with an unconditional basis [G1]. Soon afterwards, we managed to use the H.I. property to show that the complex version of $X$ gives another example. Later, we were able to pass to the real case, so $X$ itself is a counterexample to the hyperplane problem by virtue of being a H.I. space.

In fact, the space of operators on $X$ is very small: every bounded linear operator on $X$ can be written as $\lambda \operatorname{Id}+S$, where $S$ is a strictly singular operator. A question we have not answered is whether there exists a space on which every bounded linear operator is of the form $\lambda \mathrm{Id}+K$ for a compact operator $K$. We do not even know whether our space has that property, though it seems unlikely.

The rest of this paper is divided into five sections. The first concerns the notion of an asymptotic set, which is a definition of great importance for this 
problem but which arises most naturally in the context of the distortion problem, about which we shall have more to say later. In particular, we give a criterion for a space to have an equivalent norm in which it contains no $C$-unconditional basic sequence.

The second section is about a remarkable space constructed by Schlumprecht, of which our example is a development. We show that, for every $C$, his space satisfies our criterion and therefore can be renormed so as not to contain a $C$-unconditional basic sequence.

The third section contains the definition of $X$ and a proof that it is H.I. and therefore contains no unconditional basic sequence and ends with the (easy) proof that $X$ is reflexive. The fourth is about consequences of this property, especially the existence of very few operators on a complex space having it. This section does not depend on our particular construction and can therefore be read independently of the first three. The final section concerns the passage to the real case of the results of the previous one and also contains a useful strengthening of the statement that every operator on our space is the sum of a multiple of the identity and a strictly singular operator.

We are very grateful to W. B. Johnson for his influence on this paper. As we have mentioned, his observation that our space is H.I. lies at the heart of all its interesting properties. He also explained to us much simpler arguments for some of the consequences of this property. We also thank P. G. Casazza and R. G. Haydon for interesting conversations and suggestions about the problems solved here.

For the rest of this paper we shall use the words "space" and "subspace" to refer to infinite-dimensional spaces and subspaces. Similarly a basis will always be assumed to be infinite.

\section{AsYMPtotic SETS}

Let $X$ be a normed space and let $S(X)$ be its unit sphere. We shall say that a subset $A \subset S(X)$ is asymptotic if $A \cap S(Y) \neq \varnothing$ for every infinite-dimensional (not necessarily closed) subspace $Y \subset X$. A key observation for this paper is that, if a space $X$ contains infinitely many asymptotic sets that are all well disjoint from one another, then these can be used to construct an equivalent norm on $X$ such that no sequence is $C$-unconditional in this norm. In this section, we shall make that statement precise and prove it. The approach also occurred naturally (for the second author in particular) as a generalization of [MR]. It will underlie most of the arguments of this paper.

Let $A_{1}, A_{2}, \ldots$ be a sequence of subsets of the unit sphere of a normed space $X$, and let $A_{1}^{*}, A_{2}^{*}, \ldots$ be a sequence of subsets of the unit ball of $X^{*}$. (It is slightly more convenient in applications to take the ball rather than the sphere.) We shall say that $A_{1}, A_{2}, \ldots$ and $A_{1}^{*}, A_{2}^{*}, \ldots$ are an asymptotic biorthogonal system with constant $\delta$ if the following conditions hold.

(i) For every $n \in \mathbb{N}$, the set $A_{n}$ is asymptotic.

(ii) For every $n \in \mathbb{N}$ and every $x \in A_{n}$, there exists $x^{*} \in A_{n}^{*}$ such that $x^{*}(x)>1-\delta$.

(iii) For every $n, m \in \mathbb{N}$ with $n \neq m$, every $x \in A_{n}$, and every $x^{*} \in A_{m}^{*}$, $\left|x^{*}(x)\right|<\delta$. 
Under these circumstances, we shall say that $X$ contains an asymptotic biorthogonal system. The definition is not interesting if $\delta>1 / 2$ since one may take $A_{n}=S(X)$ and $A_{n}^{*}=\frac{1}{2} B\left(X^{*}\right)$ for every $n$. On the other hand, if $\delta<1 / 2$, it is not at all obvious that any Banach space contains an asymptotic biorthogonal system with constant $\delta$. We shall see later, however, that this is not as rare a phenomenon as it might seem.

Note that the $A_{n}$ are separated in the following sense. If $n \neq m, x \in A_{n}$ and $y \in A_{m}$ then there exists $x^{*} \in A_{n}^{*}$ such that $x^{*}(x)>1-\delta$ and $\left|x^{*}(y)\right|<\delta$. Since $A_{n}^{*} \subset B\left(X^{*}\right)$, it follows that $\|x-y\|>1-2 \delta$.

In practice, one usually obtains a stronger property. There is a sequence of constants $\left(\delta_{n}\right)_{n=1}^{\infty}$ tending to zero such that one can replace $\delta$ in conditions (ii) and (iii) by $\delta_{n}$ and $\delta_{\min \{m, n\}}$ respectively. This we call an asymptotic biorthogonal sequence with vanishing constant.

The main result of this section is the following theorem.

Theorem 2. Let $0<\delta<1 / 36$, and let $X$ be a separable normed space containing an asymptotic biorthogonal system with constant $\delta$. Then there is an equivalent norm on $X$ such that no sequence is $1 / \sqrt{36 \delta}$-unconditional.

Proof. Let $\|$.$\| be the original norm on X$, and let $A_{1}, A_{2}, \ldots$ and $A_{1}^{*}, A_{2}^{*}, \ldots$ be the asymptotic biorthogonal system with constant $\delta$. For each $n$ let $Z_{n}^{*}$ be a countable subset of $A_{n}^{*}$ such that for every $x \in A_{n}$ there exists $x^{*} \in Z_{n}^{*}$ with $x^{*}(x)>1-\delta$. Let $Z^{*}=\bigcup_{n=1}^{\infty} Z_{n}^{*}$. Next, let $\sigma$ be an injection to the natural numbers from the collection of finite sequences of elements of $Z^{*}$.

We shall now define a collection of functionals which we call special functionals. A special sequence of functionals of length $r$ is a sequence of the form $z_{1}^{*}, z_{2}^{*}, \ldots, z_{r}^{*}$, where $z_{1}^{*} \in Z_{1}^{*}$ and, for $1 \leqslant i<r, z_{i+1}^{*} \in Z_{\sigma\left(z_{1}^{*}, \ldots, z_{i}^{*}\right)}^{*}$. A special functional of length $r$ is simply the sum of a special sequence of length $r$. We shall let $\Gamma_{r}$ stand for the collection of special functionals of length $r$.

We can now define an equivalent norm on $X$. Let $r=\left\lfloor\delta^{-1 / 2}\right\rfloor$, and define a norm ||$|\cdot|||$ by

$$
\|\| x\|=\| x \| \vee r \max \left\{\left|z^{*}(x)\right|: z^{*} \in \Gamma_{r}\right\} .
$$

Let $x_{1}, x_{2}, \ldots$ be any sequence of linearly independent vectors in $X$. We shall show that it is not $(r-1) / 4$-unconditional in the norm $\||\cdot|\|$. We shall do this by constructing a sequence of vectors $z_{1}, \ldots, z_{r}$, generated by $x_{1}, x_{2}, \ldots$ and disjointly supported with respect to these vectors, with the property that

$$
(r-1)\left\||| \sum_{i=1}^{r}(-1)^{i} z_{i}\right\|\left|<4\left\|\mid \sum_{i=1}^{r} z_{i}\right\| \| .\right.
$$

This will obviously prove the theorem, since $(r-1) / 4>1 / \sqrt{36 \delta}$.

Let $X_{1}$ be the algebraic subspace generated by $\left(x_{i}\right)_{i=1}^{\infty}$. Since $A_{1}$ is an asymptotic set, we can find $z_{1} \in A_{1} \cap X_{1}$. This implies that $z_{1}$ has norm 1 and is generated by finitely many of the $x_{i}$. Next we can find $z_{1}^{*} \in Z_{1}^{*}$ such that $z_{1}^{*}\left(z_{1}\right)>1-\delta$. Now let $X_{2}$ be the algebraic subspace generated by all the $x_{i}$ 
not used to generate $z_{1}$. Since $A_{\sigma\left(z_{1}^{*}\right)}$ is asymptotic, we can find $z_{2} \in A_{\sigma\left(z_{1}^{*}\right)} \cap X_{2}$ of norm 1. We can then find $z_{2}^{*} \in Z_{\sigma\left(z_{1}^{*}\right)}^{*}$ such that $z_{2}^{*}\left(z_{2}\right)>1-\delta$.

Continuing this process, we obtain sequences $z_{1}, \ldots, z_{r}$ and $z_{1}^{*}, \ldots, z_{r}^{*}$ with the following properties. First, $\left\|z_{i}\right\|=1$ for each $i$. Second, $z_{i+1}^{*} \in$ $Z_{\sigma\left(z_{1}^{*}, \ldots, z_{i}^{*}\right)}^{*}$ for each $i$ (i.e., $z_{1}^{*}, \ldots, z_{r}^{*}$ is a special sequence of length $r$ ). Third, $z_{i}^{*}\left(z_{i}\right)>1-\delta$ for each $i$. Fourth, since $\sigma$ is an injection, the $z_{i}^{*}$ belong to different $A_{n}^{*}$ 's, so $\left|z_{i}^{*}\left(z_{j}\right)\right|<\delta$ when $i \neq j$.

Let us now estimate $\left\|\left|\sum_{i=1}^{r} z_{i} \|\right|\right.$. Since $z_{1}^{*}, \ldots, z_{r}^{*}$ is a special functional of length $r$, the norm is at least

$$
\begin{aligned}
r\left(\sum_{i=1}^{r} z_{i}^{*}\right)\left(\sum_{i=1}^{r} z_{i}\right) & >r((1-\delta) r-\delta r(r-1)) \\
& \geqslant r(r-1) .
\end{aligned}
$$

On the other hand, if $\left(w_{i}^{*}\right)_{i=1}^{r}$ is any special sequence of length $r$, let $t$ be maximal such that $w_{i}^{*}=z_{i}^{*}$ (or zero if $w_{1}^{*} \neq z_{1}^{*}$ ). Then

$$
\left|\sum_{i=1}^{r}(-1)^{i} w_{i}^{*}\left(z_{i}\right)\right| \leqslant\left|\sum_{i=1}^{t}(-1)^{i} w_{i}^{*}\left(z_{i}\right)\right|+\left|w_{t+1}^{*}\left(z_{t+1}\right)\right|+\sum_{i=t+2}^{r}\left|w_{i}^{*}\left(z_{i}\right)\right| .
$$

Since $\sigma$ is an injection, $w_{i}^{*}$ and $z_{j}^{*}$ are chosen from different sets $A_{n}^{*}$ whenever $i \neq j$ or $i=j>t+1$. By property (iii) this tells us that $\left|w_{i}^{*}\left(z_{j}\right)\right|<\delta$. In particular, $\sum_{i=t+2}^{r}\left|w_{i}^{*}\left(z_{i}\right)\right|<\delta r$. When $i \leq t$ we know that $1-\delta<w_{i}^{*}\left(z_{i}\right) \leqslant 1$, so $\left|\sum_{i=1}^{t}(-1)^{i} w_{i}^{*}\left(z_{i}\right)\right| \leqslant 1+\delta t / 2$. It follows that

$$
\left|\sum_{i=1}^{r}(-1)^{i} w_{i}^{*}\left(z_{i}\right)\right| \leqslant 1+\delta r / 2+1+\delta r \leqslant 2(1+\delta r) .
$$

We also know that $\sum_{i \neq j}\left|w_{i}^{*}\left(z_{j}\right)\right| \leqslant \delta r(r-1)$. Finally, by the triangle inequality, $\left\|\sum_{i=1}^{r}(-1)^{i} z_{i}\right\| \leqslant r$.

Putting all these estimates together, we find that

$$
\left\|\left|\sum_{i=1}^{r}(-1)^{i} z_{i} \|\right| \leqslant r(2(1+\delta r)+\delta r(r-1))<4 r,\right.
$$

from which it follows that the basic sequence $x_{1}, x_{2}, \ldots$ was not $(r-1) / 4-$ unconditional in the equivalent norm.

With a little more care one can increase the best unconditional constant from roughly $\delta^{-1 / 2}$ to roughly $\delta^{-1}$, but some of the details of this would obscure the main point of the proof. It also does not seem to be necessary in applications. Indeed, it is not known whether there exists a space containing an asymptotic biorthogonal system for some (nontrivial) $\delta$ but not with vanishing constant. In the next section, we examine a space that does contain one with vanishing constant.

\section{SCHLUMPRECHT'S SPACE}

A space $(Y,\|\|$.$) is said to be \lambda$-distortable if there exists an equivalent norm \|\|$\cdot \|$ on $Y$ such that for every subspace $Z \subset Y$ the quantity 


$$
\sup \{\|\mid\| y\|/\| z\|:\| y\|=\| z \|=1\}
$$

is at least $\lambda$. A space is distortable if it is $\lambda$-distortable for some $\lambda>1$. It was a famous open problem, known as the distortion problem, whether $\ell_{2}$ was distortable. This is equivalent to asking whether every space isomorphic to $\ell_{2}$ contains a subspace almost isometric to $\ell_{2}$. The problem appears as problem 2.e. 2 of [LT] but was well known by then. Some related results were proved in [LP].

A few months after the results in this paper were obtained, the distortion problem was also solved, by Odell and Schlumprecht [OS]. Actually a stronger statement is proved in [OS], namely, that $\ell_{2}$ contains an asymptotic biorthogonal system with any constant $\delta>0$ or even with vanishing constant. This implies that $\ell_{2}$ can be renormed so as not to contain a $C$-unconditional basic sequence. However, we shall consider in this section a space constructed by Schlumprecht [S1]. This space was the first known example of a space that is $\lambda$-distortable for every $\lambda$. The main result of this section is that it contains an asymptotic biorthogonal system for any $\delta$. In proving this, we shall use very little more than what was already proved by Schlumprecht in order to show that it is arbitrarily distortable.

First, let us give the definition of Schlumprecht's space. He defines a class of functions $f:[1, \infty) \rightarrow[1, \infty)$, which we shall call $\mathscr{F}$, as follows. The function $f$ is a member of $\mathscr{F}$ if it satisfies the following five conditions:

(i) $f(1)=1$ and $f(x)<x$ for every $x>1$.

(ii) $f$ is strictly increasing and tends to infinity.

(iii) $\lim _{x \rightarrow \infty} x^{-q} f(x)=0$ for every $q>0$.

(iv) The function $x / f(x)$ is concave and nondecreasing.

(v) $f(x y) \leqslant f(x) f(y)$ for every $x, y \geqslant 1$.

It is easily verified that $f(x)=\log _{2}(x+1)$ satisfies these conditions, as does the function $\sqrt{f(x)}$. Note also that some of the conditions above are redundant. In particular, one can deduce that $f(x)$ and $x / f(x)$ are strictly increasing from the other conditions.

Schlumprecht's space is a Tsirelson-type construction in that it is defined inductively. As with an earlier construction due to Tzafriri, the admissibility condition used in Tsirelson's space [T] is not needed (see [CS]). Before giving the definition, let us fix some notation.

Let $c_{00}$ be the space of sequences of scalars all but finitely many of which are zero. We shall let $\mathbf{e}_{1}, \mathbf{e}_{2}, \ldots$ stand for the unit vector basis of this vector space. If $E \subset \mathbb{N}$ then we shall also use the letter $E$ for the projection from $c_{00}$ to $c_{00}$ defined by $E\left(\sum_{i=1}^{\infty} a_{i} \mathbf{e}_{i}\right)=\sum_{i \in E} a_{i} \mathbf{e}_{i}$. If $E, F \subset \mathbb{N}$ then we write $E<F$ to mean that $\max E<\min F$, and if $k \in \mathbb{N}$ and $E \subset \mathbb{N}$ then we write $k<E$ to mean $k<\min E$. The support of a vector $x=\sum_{i=1}^{\infty} x_{i} \mathbf{e}_{i} \in c_{00}$ is the set of $i \in \mathbb{N}$ for which $x_{i} \neq 0$. An interval of integers is a subset of $\mathbb{N}$ of the form $\{a, a+1, \ldots, b\}$ for some $a, b \in \mathbb{N}$. We shall also define the range of a vector, written $\operatorname{ran}(x)$, to be the smallest interval containing its support. We shall write $x<y$ to mean $\operatorname{ran}(x)<\operatorname{ran}(y)$. If $x_{1}<\cdots<x_{n}$, we shall say that $x_{1}, \ldots, x_{n}$ are successive. 
Now let $f(x)$ be the function $\log _{2}(x+1)$ as above. If $x \in c_{00}$, its norm in Schlumprecht's space is defined by the equation

$$
\|x\|=\|x\|_{\infty} \vee \sup f(N)^{-1} \sum_{i=1}^{N}\left\|E_{i} x\right\|
$$

where the supremum runs over all integers $N \geqslant 2$ and all sequences of sets $E_{1}<\cdots<E_{N}$. Note that this definition, although apparently circular, in fact determines a unique norm. Note also that the standard basis of $c_{00}$ is oneunconditional in this norm, so there is no difference if we assume that all the sequences $E_{1}<\cdots<E_{N}$ are sequences of intervals. Later in the paper it will make a great difference, and we now adopt the convention that all such sequences mentioned are sequences of intervals.

We now prove various lemmas about this space. As we have already said, they are essentially due to Schlumprecht [S1, S2] but are stated here in slightly greater generality so that they can be applied to our space in the main part of this paper.

Let $\mathscr{X}$ be the set of normed spaces of the form $X=\left(c_{00},\|\|.\right)$ such that $\left(\mathbf{e}_{i}\right)_{i=1}^{\infty}$ is a normalized monotone basis of $X$. If $f \in \mathscr{F}, X \in \mathscr{X}$ and every $x \in X$ satisfies the inequality

$$
\|x\| \geqslant \sup \left\{f(N)^{-1} \sum_{i=1}^{N}\left\|E_{i} x\right\|: N \in \mathbb{N}, E_{1}<\cdots<E_{N}\right\}
$$

then we shall say that $X$ satisfies a lower $f$-estimate. (It is important that, in the supremum above, the $E_{i}$ are intervals.) Note that this implies that $\|E x\| \leqslant\|x\|$ for every interval $E$ and vector $x$, so the standard basis of a space with a lower $f$-estimate is automatically bimonotone.

Given a space $X \in \mathscr{X}$ and a vector $x \in X$, we shall say that $x$ is an $\ell_{1+}^{n}$-average with constant $C$ if $\|x\|=1$ and $x=\sum_{i=1}^{n} x_{i}$ for some sequence $x_{1}<\cdots<x_{n}$ of nonzero elements of $X$ such that $\left\|x_{i}\right\| \leqslant C n^{-1}$ for each $i$. An $\ell_{1+}^{n}$-vector is any positive multiple of an $\ell_{1+}^{n}$-average. In other words, a vector $x$ is an $\ell_{1+}^{n}$-vector with constant $C$ if it can be written $x=x_{1}+\cdots+x_{n}$, where $x_{1}<\cdots<x_{n}$, the $x_{i}$ are nonzero, and $\left\|x_{i}\right\| \leqslant C n^{-1}\|x\|$ for every $i$.

Finally, by a block basis in a space $X \in \mathscr{X}$ we mean a sequence $x_{1}, x_{2}, \ldots$ of successive nonzero vectors in $X$ (note that such a sequence must be a basic sequence), and by a block subspace of a space $X \in \mathscr{X}$ we mean a subspace generated by a block basis.

Lemma 3. Let $f \in \mathscr{F}$, and let $X \in \mathscr{X}$ satisfy a lower $f$-estimate. Then, for every $n \in \mathbb{N}$ and every $C>1$, every block subspace $Y$ of $X$ contains an $\ell_{1+}^{n}$-average with constant $C$.

Proof. Suppose the result is false. Let $k$ be an integer such that $k \log C>$ $\log f\left(n^{k}\right)$ (such an integer exists because of property (iii) in the definition of $\mathscr{F}$ ), let $N=n^{k}$, let $x_{1}<\cdots<x_{N}$ be any sequence of successive norm-1 vectors in $Y$, and let $x=\sum_{i=1}^{N} x_{i}$. For every $0 \leqslant i \leqslant k$ and every $1 \leqslant j \leqslant$ $n^{k-i}$, let $x(i, j)=\sum_{t=(j-1) n^{i}+1}^{j n^{i}} x_{t}$. Thus $x(0, j)=x_{j}, x(k, 1)=x$, and, 
for $1 \leqslant i \leqslant k$, each $x(i, j)$ is a sum of $n$ successive $x(i-1, j)$ 's. By our assumption, no $x(i, j)$ is an $\ell_{1+}^{n}$-vector with constant $C$. It follows easily by induction that $\|x(i, j)\| \leqslant C^{-i} n^{i}$ and, in particular, that $\|x\| \leqslant C^{-k} n^{k}=$ $C^{-k} N$. However, it follows from the fact that $X$ satisfies a lower $f$-estimate that $\|x\| \geqslant N f(N)^{-1}$. This is a contradiction, by choice of $k$.

Lemma 4. Let $M, N \in \mathbb{N}$ and $C \geqslant 1$, let $X \in \mathscr{X}$, let $x \in X$ be an $\ell_{1+}^{N}$-vector with constant $C$, and let $E_{1}<\cdots<E_{M}$ be a sequence of intervals. Then

$$
\sum_{j=1}^{M}\left\|E_{j} x\right\| \leqslant C(1+2 M / N)\|x\| .
$$

Proof. For convenience, let us normalize so that $\|x\|=N$ and $x=\sum_{1=1}^{N} x_{i}$, where $x_{1}<\cdots<x_{N}$ and $\left\|x_{i}\right\| \leqslant C$ for each $i$. Given $j$, let $A_{j}$ be the set of $i$ such that $\operatorname{supp}\left(x_{i}\right) \subset E_{j}$ and let $B_{j}$ be the set of $i$ such that $E_{j}\left(x_{i}\right) \neq 0$. By the triangle inequality and the fact that the basis is bimonotone,

$$
\left\|E_{j} x\right\| \leqslant\left\|\sum_{i \in B_{j}} x_{i}\right\| \leqslant C\left(\left|A_{j}\right|+2\right) .
$$

Since $\sum_{j=1}^{M}\left|A_{j}\right| \leqslant N$, we get

$$
\sum_{j=1}^{M}\left\|E_{j} x\right\| \leqslant C(N+2 M),
$$

which gives the result, because of our normalization.

In order to state the next lemma, we shall need some more definitions. The first is a technicality. If $f \in \mathscr{F}$, let $M_{f}: \mathbb{R} \rightarrow \mathbb{R}$ be defined by $M_{f}(x)=$ $f^{-1}\left(36 x^{2}\right)$.

The next definition is of great importance in this paper. We shall say that a sequence $x_{1}<\cdots<x_{N}$ is a rapidly increasing sequence of $\ell_{1+}$-averages, or R.I.S., for $f$ of length $N$ with constant $1+\epsilon$ if $x_{k}$ is an $\ell_{1+}^{n_{k}}$-average with constant $1+\epsilon$ for each $k, n_{1} \geqslant 2(1+\epsilon) M_{f}\left(N / \epsilon^{\prime}\right) / \epsilon^{\prime} f^{\prime}(1)$, and

$$
\frac{\epsilon^{\prime}}{2} f\left(n_{k}\right)^{1 / 2} \geqslant\left|\operatorname{supp}\left(x_{k-1}\right)\right|
$$

for $k=2, \ldots, N$. Here $f^{\prime}(1)$ is the right derivative of $f$ at 1 and $\epsilon^{\prime}$ is a useful notation for $\min \{\epsilon, 1\}$, which we shall use throughout the section. Obviously there is nothing magic about the exact conditions in this definition. The important point is that the $n_{k}$ 's increase fast enough, the speed depending on the sizes of the supports of the earlier $x_{j}$ 's. It will sometimes be convenient to call a vector a R.I.S.-vector if it is a nonzero multiple of the sum of a R.I.S.

We make one further definition. A functional $x^{*}$ is an $(M, g)$-form if $\left\|x^{*}\right\| \leqslant 1$ and $x^{*}=\sum_{j=1}^{M} x_{j}^{*}$ for some sequence $x_{1}^{*}<\cdots<x_{M}^{*}$ of successive functionals such that $\left\|x_{j}^{*}\right\| \leqslant g(M)^{-1}$ for each $j$. 
Lemma 5. Let $f, g \in \mathscr{F}$, let $g \geqslant f^{1 / 2}$, and let $X \in \mathscr{Z}$ satisfy a lower $f$ estimate. Let $\epsilon>0$, let $x_{1}, \ldots, x_{N}$ be a R.I.S. in $X$ for $f$ with constant $1+\epsilon$, and let $x=\sum_{i=1}^{N} x_{i}$. Let $M \geqslant M_{f}\left(N / \epsilon^{\prime}\right)$, let $x^{*}$ be an $(M, g)$-form, and let $E$ be any interval. Then $\left|x^{*}(E x)\right| \leqslant 1+\epsilon+\epsilon^{\prime}$.

Proof. If $x^{*}$ is an $(M, g)$-form then so is $E x^{*}$ for any interval $E$. Since $x^{*}(E x)=\left(E x^{*}\right)(x)$, we can forget about the interval $E$ in the statement of the lemma. For each $i$, let $n_{i}$ be maximal such that $x_{i}$ is an $\ell_{1+}^{n_{i}}$-average with constant $1+\epsilon$. Let us also write $x^{*}=\sum_{j=1}^{M} x_{j}^{*}$ in the obvious way, and set $E_{j}=\operatorname{ran}\left(x_{j}^{*}\right)$. We first obtain three easy estimates for $\left|x^{*}\left(x_{i}\right)\right|$. Since $\left\|x^{*}\right\| \leqslant$ 1 , we obviously have $\left|x^{*}\left(x_{i}\right)\right| \leqslant 1$. Then, since $\left\|x_{j}^{*}\right\| \leqslant g(M)^{-1} \leqslant f(M)^{-1 / 2}$, we have $\left|x^{*}\left(x_{i}\right)\right| \leqslant f(M)^{-1 / 2} \sum_{j=1}^{M}\left\|E_{j} x_{i}\right\|$. By our assumption about $X$, this is at most $f(M)^{-1 / 2} f\left(\left|\operatorname{supp}\left(x_{i}\right)\right|\right)$, and by Lemma 4 it is at most $(1+\epsilon) \times$ $\left(1+2 M n_{i}^{-1}\right) f(M)^{-1 / 2}$.

Let $t$ be maximal such that $n_{t} \leqslant M$. Then, if $i<t$, we have $f\left(\left|\operatorname{supp}\left(x_{i}\right)\right|\right) \leqslant$ $2^{i-t+1} f\left(\left|\operatorname{supp}\left(x_{t-1}\right)\right|\right)$, and also

$$
f\left(\left|\operatorname{supp}\left(x_{t-1}\right)\right|\right) \leqslant\left(\epsilon^{\prime} / 2\right) f\left(n_{t}\right)^{1 / 2} \leqslant\left(\epsilon^{\prime} / 2\right) f(M)^{1 / 2} .
$$

Using this and the other two estimates above, we obtain

$$
\begin{aligned}
\left|x^{*}(x)\right| & \leqslant \sum_{i=1}^{N}\left|x^{*}\left(x_{i}\right)\right| \leqslant \epsilon^{\prime}+1+3(1+\epsilon)(N-t) f(M)^{-1 / 2} \\
& \leqslant 1+\epsilon^{\prime}+3(1+\epsilon) N\left(\epsilon^{\prime} / 6 N\right) \\
& =1+\epsilon^{\prime}+\left(\epsilon^{\prime} / 2\right)(1+\epsilon) \leqslant 1+\epsilon+\epsilon^{\prime}
\end{aligned}
$$

as stated.

Corollary 6. Let $f, X, \epsilon, M, x_{1}, \ldots, x_{N}$, and $x$ be as in Lemma 5, and let $E_{1}<\cdots<E_{M}$. Then

$$
f(M)^{-1} \sum_{i=1}^{M}\left\|E_{i} x\right\| \leqslant 1+\epsilon+\epsilon^{\prime} .
$$

Proof. Let $x_{j}^{*}$ be a support functional of $E_{j} x$, and let $x^{*}=f(M)^{-1} \sum_{i=1}^{M} x_{i}^{*}$. Then $\left\|x^{*}\right\| \leqslant 1$ because $X$ satisfies a lower $f$-estimate. It follows that $x^{*}$ is an $(M, f)$-form, so we can apply Lemma 5 with $g=f$.

We now introduce a further convenient definition. Let $x_{1}<\cdots<x_{N}$ be a R.I.S. for $f$ with constant $1+\epsilon$ for some $f \in \mathscr{F}$ and some $\epsilon>0$. For each $i$, let $n_{i}$ be maximal such that $x_{i}$ is an $\ell_{1+}^{n_{i}}$-average with constant $1+\epsilon$, and let us write it out as $x_{i}=x_{i 1}+\cdots+x_{i n_{i}}$, where $\left\|x_{i j}\right\| \leqslant(1+\epsilon) n_{i}^{-1}$ for each $j$. Given an interval $E \subset \mathbb{N}$, let $i=i_{E}$ and $j=j_{E}$ be respectively minimal and maximal 
such that $E x_{i}$ and $E x_{j}$ are nonzero, and let $r=r_{E}$ and $s=s_{E}$ be respectively minimal and maximal such that $E x_{i r}$ and $E x_{j s}$ are nonzero. Define the length $\lambda(E)$ of the interval $E$ to be $j_{E}-i_{E}+\left(s_{E} / n_{j_{E}}\right)-\left(r_{E} / n_{i_{E}}\right)$. Thus the length of $E$ is the number of $x_{i}$ 's contained in $E$, allowing for fractional parts. It is easy to check that, if $E_{1}<\cdots<E_{M}$ and $E=\bigcup E_{i}$, then $\sum \lambda\left(E_{i}\right) \leqslant \lambda(E)$. Obviously this definition depends completely on the R.I.S., but it will always be clear from the context which R.I.S. is being considered.

The next lemma is the most important for our purposes.

Lemma 7. Let $f, g \in \mathscr{F}$ with $g \geqslant \sqrt{f}$, let $X \in \mathscr{X}$ satisfy a lower f-estimate, let $\epsilon>0$, let $x_{1}<\cdots<x_{N}$ be a R.I.S. in $X$ for $f$ with constant $1+\epsilon$, and let $x=\sum_{i=1}^{N} x_{i}$. Suppose that

$$
\|E x\| \leqslant \sup \left\{\left|x^{*}(E x)\right|: M \geqslant 2, x^{*} \text { is an }(M, g) \text {-form }\right\}
$$

for every interval $E$ of length at least 1 . Then $\|x\| \leqslant\left(1+\epsilon+\epsilon^{\prime}\right) N g(N)^{-1}$.

Proof. It follows from the triangle inequality that $\|E x\| \leqslant(1+\epsilon)\left(\lambda(E)+n_{1}^{-1}\right)$. If $\lambda(E) \geqslant(1+\epsilon) / \epsilon^{\prime} n_{1}$ then we get $\|E x\| \leqslant\left(1+\epsilon+\epsilon^{\prime}\right) \lambda(E)$. Let $G$ be defined by $G(x)=x$ when $0 \leqslant x \leqslant 1$ and by $G(x)=x g(x)^{-1}$ when $x \geqslant 1$. Recall that, because of the properties of the set $\mathscr{F}, G$ is concave and increasing on $[1, \infty)$ and satisfies $G(x y) \geqslant G(x) G(y)$ for every $x, y$ in the same interval. It is easy to check that these properties are still true on the whole of $\mathbb{R}_{+}$. We shall show that, if $\lambda(E) \geqslant(1+\epsilon) / \epsilon^{\prime} n_{1}$, then $\|E x\| \leqslant\left(1+\epsilon+\epsilon^{\prime}\right) G(\lambda(E))$. The remarks we have just made show this when $\lambda(E) \leqslant 1$.

Let us suppose then that $E$ is a minimal interval of length at least $(1+\epsilon) / \epsilon^{\prime} n_{1}$ for which the inequality fails. We know that $\lambda(E) \geqslant 1$. We also know that there exists some $(M, g)$-form $x^{*}=\sum_{i=1}^{M} x_{i}^{*}$ such that $\|E x\| \leqslant\left|x^{*}(E x)\right|$. By Lemma 5, we must have $M \leqslant M_{f}\left(N / \epsilon^{\prime}\right)$, or the inequality would not fail for $E$. Letting $E_{i}=E \cap \operatorname{ran}\left(x_{i}^{*}\right)$, we have

$$
\|E x\| \leqslant g(M)^{-1} \sum_{i=1}^{M}\left\|E_{i} x\right\|
$$

by the definition of an $(M, g)$-form.

Let $\lambda_{i}=\lambda\left(E_{i}\right)$ for each $i$. Since $M \geqslant 2$, we may assume that none of the intervals $E_{i}$ are equal to $E$. For each $i$ we either have $\lambda_{i} \leqslant(1+\epsilon) / \epsilon^{\prime} n_{1}$ or, by the minimality of $E$, that $\left\|E_{i} x\right\| \leqslant\left(1+\epsilon+\epsilon^{\prime}\right) G\left(\lambda_{i}\right)$. Let $A$ be the set of $i$ with the first property, and let $B$ be the complement of $A$. Let $k$ be the cardinality of $A$. It is clear that $k<M$, using the R.I.S. condition.

Since $G$ is a concave and nondecreasing function and $\sum \lambda_{i} \leqslant \lambda$, Jensen's inequality gives us that

$$
\begin{aligned}
\sum_{i \in B}\left\|E_{i} x\right\| & \leqslant\left(1+\epsilon+\epsilon^{\prime}\right) \sum_{i \in B} G\left(\lambda_{i}\right) \\
& \leqslant\left(1+\epsilon+\epsilon^{\prime}\right)(M-k) G(\lambda /(M-k)) .
\end{aligned}
$$


It follows that

$$
\begin{aligned}
\|E x\| & \leqslant M^{-1} G(M)\left[\left(1+\epsilon+\epsilon^{\prime}\right)(M-k) G(\lambda /(M-k))\right. \\
& \left.+(1+\epsilon)\left(1+\epsilon+\epsilon^{\prime}\right) k / \epsilon^{\prime} n_{1}\right] \\
& \leqslant\left(1+\epsilon+\epsilon^{\prime}\right)\left[(1-k / M) G(M) G(\lambda /(M-k))+(1+\epsilon) k / \epsilon^{\prime} n_{1}\right] \\
& \leqslant\left(1+\epsilon+\epsilon^{\prime}\right)\left[(1-k / M) G\left((1-k / M)^{-1} \lambda\right)+(1+\epsilon) k / \epsilon^{\prime} n_{1}\right] .
\end{aligned}
$$

Let $G^{\prime}(1)$ be the right derivative of $G$ at 1 . Since $G$ is a concave function, we have the easy inequality

$$
(1-t) G\left(\frac{\lambda}{1-t}\right)+t\left(G(1)-G^{\prime}(1)\right) \leqslant G(\lambda)
$$

for every $0 \leqslant t<1$ and $\lambda \geqslant 1$. Also, $G(1)-G^{\prime}(1)=1-G^{\prime}(1)=g^{\prime}(1)$, and, since $g \geqslant \sqrt{f}$, we have $g^{\prime}(1) \geqslant f^{\prime}(1) / 2>0$.

By the definition of R.I.S. we have $n_{1} \geqslant 2(1+\epsilon) M_{f}\left(N / \epsilon^{\prime}\right) / \epsilon^{\prime} f^{\prime}(1)$. It follows that

$(1+\epsilon) k / \epsilon^{\prime} n_{1} \leqslant(k / M)\left((1+\epsilon) M_{f}\left(N / \epsilon^{\prime}\right) / \epsilon^{\prime} n_{1}\right) \leqslant(k / M)\left(f^{\prime}(1) / 2\right) \leqslant(k / M) g^{\prime}(1)$.

Hence, by the inequality above with $t=k / M$, we have

$$
\begin{aligned}
(1+\epsilon & \left.+\epsilon^{\prime}\right)\left[(1-k / M) G\left((1-k / M)^{-1} \lambda\right)+(1+\epsilon) k / \epsilon^{\prime} n_{1}\right] \\
& \leqslant\left(1+\epsilon+\epsilon^{\prime}\right)\left((1-t) G\left(\frac{\lambda}{1-t}\right)+t^{\prime}(1)\right) \\
& \leqslant\left(1+\epsilon+\epsilon^{\prime}\right) G(\lambda),
\end{aligned}
$$

contradicting our assumption about the interval $E$ and proving the lemma.

It is now easy to construct an asymptotic biorthogonal system in Schlumprecht's space. Let $\delta \in(0,1)$ and let $N_{1}<N_{2}<\cdots$ be a sequence of squares satisfying $f\left(N_{1}\right) / N_{1}<\delta / 2, f\left(N_{1}\right)>8 \delta^{-1}$, and $N_{j}>M_{f}\left(2 N_{j-1}\right)$ for all $j>1$. Let $A_{k}$ be the set of norm-1 vectors of the form $x=\sum_{i=1}^{N_{k}} x_{i}$ where $x_{1}, \ldots, x_{N_{k}}$ is a multiple of a R.I.S. with constant $1+\delta / 2$. Because Schlumprecht's space satisfies a lower $f$-estimate, we know that the multiple is at most $f\left(N_{k}\right) N_{k}^{-1}$. Let $A_{k}^{*}$ be the set of functionals of the form $f\left(N_{k}\right)^{-1} \sum_{i=1}^{N_{k}} x_{i}^{*}$ where $x_{1}^{*}<\cdots<x_{N_{k}}^{*}$ and $\left\|x_{i}^{*}\right\| \leqslant 1$ for each $i$. It is clear that the sets $A_{k}$ are asymptotic for every $k$. If $j>k$ then, using the fact that $N_{j}>M_{f}\left(2 N_{k}\right)$, we may apply Lemma 5 with $\epsilon=1 / 2$ and $M=N_{j}$ since $y^{*}$ is clearly an $(M, f)$-form whenever $y^{*} \in A_{j}^{*}$. Because of the normalization of the R.I.S., this gives us $\left|y^{*}(x)\right| \leqslant 2 f\left(N_{k}\right) / N_{k}<\delta$ for every $y^{*} \in A_{j}^{*}$ and $x \in A_{k}$.

If $j<k$ then we know from Lemma 7 that $\left\|\sum_{i \in A} x_{i}\right\| \leqslant 2|A| f\left(N_{k}\right) / N_{k} f(|A|)$ for every subset $A$ of $\left\{1,2, \ldots, N_{k}\right\}$. If $|A| \geqslant \sqrt{N_{k}}$ then this is at most $4|A| / N_{k}$. By splitting into $\sqrt{N_{k}}$ successive pieces of this form, we find that 
$x$ is an $\ell_{1+}^{\sqrt{N_{k}}}$-average with constant 4 . By Lemma 4 we obtain that $\left|y^{*}(x)\right| \leqslant$ $f\left(N_{j}\right)^{-1} .4\left(1+2 N_{j} / \sqrt{N_{k}}\right) \leqslant 8 f\left(N_{j}\right)^{-1}<\delta$.

Finally, we know that $\|x\| \leqslant(1+\delta) N_{k} f\left(N_{k}\right)^{-1}\left\|x_{i}\right\|$ for each $i$, so if we let $x_{i}^{*}$ be a support functional of $x_{i}$ then $x^{*}=f\left(N_{k}\right)^{-1} \sum_{i=1}^{N_{k}} x_{i}^{*}$ is an element of $A_{k}^{*}$ and $x^{*}(x) \geqslant(1+\delta)^{-1}>1-\delta$. It follows that $A_{1}, A_{2}, \ldots$ and $A_{1}^{*}, A_{2}^{*}, \ldots$ form an asymptotic biorthogonal system with constant $\delta$. An obvious modification of this argument will also give an asymptotic biorthogonal system with vanishing constant.

This together with the result of the last section shows that, for every $C$, Schlumprecht's space can be renormed so as not to contain a $C$-unconditional basic sequence. Since Schlumprecht's space itself has a one-unconditional basis, it follows that it is arbitrarily distortable. This is also an easy direct consequence of the existence of an asymptotic biorthogonal system, or indeed of Lemma 7, which is what Schlumprecht used.

\section{A SPACE CONTAINING NO UNCONDITIONAL BASIC SEQUENCE}

We now come to the main result of the paper, namely, the construction of a Banach space $X$ containing no unconditional basic sequence. As we mentioned in the introduction, it was observed by W. B. Johnson that our original arguments could be modified to show that $X$ was H.I. This is what we shall actually present in this section.

The definition of the space resembles that of Schlumprecht's space, or at least it can do so. We shall give three equivalent definitions, for which we shall need a certain amount of preliminary notation.

First, let $\mathbf{Q}$ be the set of scalar sequences with finite support, rational coordinates, and maximum at most one in modulus. Let $J \subset \mathbb{N}$ be a set such that, if $m<n$ and $m, n \in J$, then $\log \log \log n \geqslant 4 m^{2}$. Let us write $J$ in increasing order as $\left\{j_{1}, j_{2}, \ldots\right\}$. We shall also assume that $f\left(j_{1}\right)>256$. (Recall that $f(x)$ is the function $\log _{2}(x+1)$.) Now let $K \subset J$ be the set $\left\{j_{1}, j_{3}, j_{5}, \ldots\right\}$, and let $L \subset \mathbb{N}$ be the set of integers $j_{2}, j_{4}, j_{6}, \ldots$.

Let $\sigma$ be an injection from the collection of finite sequences of successive elements of $\mathbf{Q}$ to $L$ such that, if $z_{1}, \ldots, z_{s}$ is such a sequence, $S=$ $\sigma\left(z_{1}, \ldots, z_{s}\right)$, and $z=\sum_{i=1}^{s} z_{i}$, then $(1 / 20) f\left(S^{1 / 40}\right)^{1 / 2} \geqslant|\operatorname{supp}(z)|$.

We shall use the injection $\sigma$ to define special functionals in an arbitrary normed space of the form $\left(c_{00},\|\|.\right)$ in much the same way that we defined them in $\S 1$. (Of course, for most spaces they are not terribly interesting.)

If $X=\left(c_{00},\|\|.\right)$ is a normed space on the finitely supported sequences and $m \in \mathbb{N}$, let $A_{m}^{*}(X)$ be the set of functionals of the form $f(m)^{-1} \sum_{i=1}^{m} f_{i}$ such that $f_{1}<\cdots<f_{m}$ and $\left\|f_{i}\right\| \leqslant 1$ for each $i$. If $k \in \mathbb{N}$, let $\Gamma_{k}^{X}$ be the set of sequences $g_{1}, \ldots, g_{k}$ such that $g_{i} \in \mathbf{Q}$ for each $i, g_{1} \in A_{j_{2 k}}^{*}(X)$ and $g_{i+1} \in A_{\sigma\left(g_{1}, \ldots, g_{i}\right)}^{*}(X)$ for each $1 \leqslant i \leqslant k-1$. We call these special sequences. Let $B_{k}^{*}(X)$ be the set of functionals of the form $f(k)^{-1 / 2} \sum_{j=1}^{k} g_{j}$ such that $\left(g_{1}, \ldots, g_{k}\right) \in \Gamma_{k}^{X}$. These are special functionals. 
Dually, if a convex set $D \subset c_{00}$ is given, we define $A_{m}(D)$ to be the set of vectors of the form $f(m)^{-1} \sum_{i=1}^{m} x_{i}$ where $x_{1}<\cdots<x_{m}$ and $x_{i} \in D$ for each $i$. Then special sequences of vectors are defined using $\sigma$ in the obvious corresponding way, and this gives us sets $B_{k}(D)$.

Our first definition of the norm is geometrical and goes via the dual space. Let $D_{0}$ be the intersection of $c_{00}$ with the unit ball of $\ell_{1}$. Once we have defined $D_{N}$, let $D_{N}^{\prime}$ be the set of vectors of the form $f(n)^{-1} \sum_{i=1}^{n} x_{i}$, where $x_{1}, \ldots, x_{n}$ are successive vectors in $D_{N}$. Let $D_{N}^{\prime \prime}$ be the set of special vectors for $D_{N}$ with lengths in $K$, that is, $D_{N}^{\prime \prime}=\bigcup_{k \in K} B_{k}\left(D_{N}\right)$. Let $D_{N}^{\prime \prime \prime}$ be the set of all vectors $E x$ where $x \in D_{N}$ and $E$ interval. Then let $D_{N+1}$ be the convex hull of the union of $D_{N}^{\prime}$, every $\lambda D_{N}^{\prime \prime}$ with $|\lambda|=1$, and $D_{N}^{\prime \prime \prime}$.

Now let $D=\bigcup_{N=0}^{\infty} D_{N}$. It is easy to see that $D$ is the smallest symmetric convex set containing $D_{0}$ that is closed under taking successive sums of the form $f(n)^{-1} \sum_{i=1}^{n} x_{i}$, taking special vectors with lengths in $K$, and under interval projections. Our space is defined by $\|x\|=\sup \{|\langle x, y\rangle|: y \in D\}$.

The second definition of the norm is as the limit of a sequence of norms. Define $X_{0}=\left(c_{00},\|\cdot\|_{0}\right)$ by $\|x\|_{0}=\|x\|_{\infty}$, and, for $N \geqslant 0$, let

$$
\begin{aligned}
\|x\|_{X_{N+1}}= & \sup \left\{f(n)^{-1} \sum_{i=1}^{n}\left\|E_{i} x\right\|_{X_{N}}: n \in \mathbb{N}, E_{1}<\cdots<E_{n}\right\} \\
& \vee \sup \left\{|g(E x)|: k \in K, g \in B_{k}^{*}\left(X_{N}\right), E \subset \mathbb{N}\right\} .
\end{aligned}
$$

Note that this is an increasing sequence of norms, because the sets $B_{k}^{*}\left(X_{N}\right)$ increase as $N$ increases (and, more generally, if $\|x\|_{Y} \leqslant\|x\|_{Z}$ for every $x \in$ $c_{00}$, then $\left.B_{k}^{*}(Y) \subset B_{k}^{*}(Z)\right)$. They are also all bounded above by the $\ell_{1}$-norm. Define $\|$.$\| by \|x\|=\lim _{N \rightarrow \infty}\|x\|_{X_{N}}$.

Finally, we give an implicit definition of the norm in the obvious way. Set

$$
\begin{aligned}
\|x\|= & \|x\|_{c_{0}} \vee \sup \left\{f(n)^{-1} \sum_{i=1}^{n}\left\|E_{i} x\right\|: 2 \leqslant n \in \mathbb{N}, E_{1}<\cdots<E_{n}\right\} \\
& \vee \sup \left\{|g(E x)|: k \in K, g \in B_{k}^{*}(X), E \subset \mathbb{N}\right\} .
\end{aligned}
$$

Recall that $E \subset \mathbb{N}$ is always an interval in these definitions. Its role is to ensure that $\left(\mathbf{e}_{i}\right)_{i=1}^{\infty}$ is a (bimonotone) normalized Schauder basis for the completion of $X$. Note also that, if we did not insist that the $E_{i}$ were intervals, then the unit vector basis of this space would trivially be unconditional. It is not hard to check that the norm given by the third definition is indeed well defined and agrees with both the previous ones.

Before getting down to analysing the space, we shall need a few simple facts about functions in the class $\mathscr{F}$ defined earlier.

We shall now introduce some convenient definitions. Let

$$
f:[1, \infty) \rightarrow[1, \infty)
$$

be a function. The (increasing) submultiplicative hull of $f$ is the function $F$ 
defined by

$$
F(x)=\inf \left\{f\left(x_{1}\right) f\left(x_{2}\right) \cdots f\left(x_{k}\right): k \in \mathbb{N}, x_{i} \geqslant 1, x_{1} \cdots x_{k} \geqslant x\right\} .
$$

The following facts are trivial. First, $F \leqslant f$. Second, $F(x y) \leqslant F(x) F(y)$. Third, if $g:[1, \infty) \rightarrow[1, \infty)$ is any nondecreasing submultiplicative function dominated by $f$ then $g$ is dominated by $F$. (That is, $F$ is the largest nondecreasing submultiplicative function dominated by $f$.)

Now let $g:[1, \infty) \rightarrow[1, \infty)$ be any function. The concave envelope of $g$ is, of course, the smallest concave function $G:[1, \infty) \rightarrow[1, \infty)$ dominating $g$, that is,

$$
G(x)=\sup \{\lambda g(y)+(1-\lambda) g(z): 0 \leqslant \lambda \leqslant 1, \lambda y+(1-\lambda) z=x\} .
$$

We now prove an easy lemma.

Lemma 8. If $g:[1, \infty) \rightarrow[1, \infty)$ is a supermultiplicative function then its concave envelope $G$ is also supermultiplicative.

Proof. Let $\epsilon>0$, and let $x_{1}, x_{2} \geqslant 1$. We shall show that $\left(G\left(x_{1}\right)-\epsilon\right) \times$ $\left(G\left(x_{2}\right)-\epsilon\right) \leqslant G\left(x_{1}\right) G\left(x_{2}\right)$, which will prove the result. First, for $i=1,2$, pick $\lambda_{i}, \mu_{i}, y_{i}$, and $z_{i}$ such that $0 \leqslant \lambda_{i} \leqslant 1, \lambda_{i}+\mu_{i}=1, \lambda_{i} y_{i}+\mu_{i} z_{i}=x_{i}$, and $\lambda_{i} g\left(y_{i}\right)+\mu_{i} g\left(z_{i}\right) \geqslant G\left(x_{i}\right)-\epsilon$.

Then

$$
\begin{aligned}
& \left(G\left(x_{1}\right)-\epsilon\right)\left(G\left(x_{2}\right)-\epsilon\right) \\
& \quad \leqslant\left(\lambda_{1} g\left(y_{1}\right)+\mu_{1} g\left(z_{1}\right)\right)\left(\lambda_{2} g\left(y_{2}\right)+\mu_{2} g\left(z_{2}\right)\right) \\
& \quad \leqslant \lambda_{1} \lambda_{2} g\left(y_{1} y_{2}\right)+\lambda_{1} \mu_{2} g\left(y_{1} z_{2}\right)+\mu_{1} \lambda_{2} g\left(z_{1} y_{2}\right)+\mu_{1} \mu_{2} g\left(z_{1} z_{2}\right) \\
& \quad \leqslant \lambda_{1} G\left(y_{1} x_{2}\right)+\mu_{1} G\left(z_{1} x_{2}\right) \leqslant G\left(x_{1} x_{2}\right)
\end{aligned}
$$

as we wanted.

Now let $K_{0} \subset K$, and let us define a function $\phi:[1, \infty) \rightarrow[1, \infty)$ as

$$
\phi(x)= \begin{cases}\left(\log _{2}(x+1)\right)^{1 / 2} & \text { if } x \in K_{0} \\ \log _{2}(x+1) & \text { otherwise }\end{cases}
$$

Let $h$ be the submultiplicative hull of $\phi$, let $H$ be the function given by $H(x)=x / h(x)$, and let $G$ be the concave envelope of $H$. Since $h$ is submultiplicative, $H$ is supermultiplicative, and so $G$ is also supermultiplicative. Now let $g(x)=x / G(x)$. Then $g$ is submultiplicative. As before, let $f$ be the function $\log _{2}(x+1)$. The easy facts about submultiplicative hulls and concave envelopes and the fact that $\sqrt{f} \in \mathscr{F}$ give that $\left(\log _{2}(x+1)\right)^{1 / 2} \leqslant g(x) \leqslant \phi(x) \leqslant$ $\log _{2}(x+1)$. It follows easily from these comments, and the remarks following the definition of $\mathscr{F}$, that $g \in \mathscr{F}$. It will be useful to extend the definition of $G$ to the whole of $\mathbb{R}_{+}$by setting $G(x)=x$ when $0 \leqslant x \leqslant 1$. It is easy to check that $G$ thus extended is still supermultiplicative and concave, as we commented in the proof of Lemma 7.

We now need to calculate $G(N)$ when $N \in J \backslash K_{0}$. In fact, we shall want slightly more than this, as is suggested by the statement of the next lemma. 
Lemma 9. If $N \in J \backslash K_{0}$ then $G(x)=x f(x)^{-1}$ for every $x$ in the interval $[\log N, \exp N]$.

Proof. Let $k, l \in K_{0} \cup\{1\}$ be maximal and minimal respectively such that $k<N$ and $l>N$, and let $(k !)^{4}<x<f^{-1}\left(f(l)^{1 / 2}\right)$. We shall show first that $h(x)=f(x)$. Recall that $h(x)$ is defined to be $\inf \left\{\phi\left(x_{1}\right) \cdots \phi\left(x_{m}\right): x_{i} \geqslant\right.$ $\left.1, x_{1} x_{2} \cdots x_{m} \geqslant x\right\}$.

Assume then that $x_{1}, \ldots, x_{m}$ are such that $h(x)=\phi\left(x_{1}\right) \cdots \phi\left(x_{m}\right)$ and $x_{1} \cdots x_{m} \geqslant x$. We may assume that $x_{i}>1$ for every $i$. We know that $\phi\left(x_{j}\right)=f\left(x_{j}\right)^{1 / 2}$ if $x_{j} \in K_{0}$ and $f\left(x_{j}\right)$ otherwise. By the submultiplicativity of $f$, there can be at most one $j$ such that $x_{j} \notin K_{0}$. Because $f(l)^{1 / 2}>f(x)$, we also know that, if $x_{j} \in K_{0}$, then $x_{j} \leqslant k$, and therefore $k>1$. Thirdly, it is not possible to find $r, s, t$ such that $x_{r}=x_{s}=x_{t} \in K_{0}$ since, for every $p \in K, f(p)^{3 / 2}>f\left(p^{3}\right)$ by our choice for $\min (K)$. Since $x>(k !)^{4}$, it is clear that at least one, and hence exactly one, $x_{j}$ is not in $K_{0}$. Let it be $x_{1}$, and assume that $m>1$. Now we know that $x_{2} x_{3} \cdots x_{m} \leqslant(k !)^{2} \leqslant x^{1 / 2}$. It follows that $x_{1} \geqslant x^{1 / 2}$ and hence that $\phi\left(x_{1}\right) \cdots \phi\left(x_{m}\right) \geqslant f\left(x^{1 / 2}\right) f(\min (K))^{1 / 2}$. Since $f$ is the function $\log _{2}(x+1)$ and $f(\min (K)) \geqslant 36$, this is greater than $f(x)$. This contradiction shows that $m=1$ and hence that $h(x)=f(x)$.

We now know that $H(x)=x f(x)^{-1}$ whenever $(k !)^{4}<x<f^{-1}\left(f(l)^{1 / 2}\right)$ and, in particular, for all $x$ in the interval $[\log \log N$, $\exp \exp N]$. It is easy to deduce from this the conclusion of the lemma. Indeed, given $x_{0}$ in the interval $[\log N, \exp N]$, we will certainly know that $G\left(x_{0}\right)=x_{0} f\left(x_{0}\right)^{-1}$ if the function given by the tangent to $x f(x)^{-1}$ at $x_{0}$ is at least $x f(x)^{-1 / 2}$ for all positive $x$ outside the interval $[\log \log N$, $\exp \exp N]$.

The equation of the tangent at $x_{0}$ is

$$
y=\frac{x_{0}}{f\left(x_{0}\right)}+\frac{1}{f\left(x_{0}\right)}\left(1-\frac{x_{0}}{\left(x_{0}+1\right) \log \left(x_{0}+1\right)}\right)\left(x-x_{0}\right) .
$$

When $x \geqslant 0$ this is certainly at least $x_{0}^{2} \log 2 /\left(x_{0}+1\right)\left(\log \left(x_{0}+1\right)\right)^{2}$, which is at least $x_{0} / 2 f\left(x_{0}\right)^{2}$. For $x_{0} \geqslant \log N$ and $x \leqslant \log \log N$ this exceeds $x f(x)^{-1 / 2}$. When $x \geqslant 2 x_{0}$ we also know that $y \geqslant x / 4 f\left(x_{0}\right)$. When $x \geqslant \exp \exp N$ the condition $x_{0} \leqslant \exp N$ is enough to guarantee that this is at least as big as $x f(x)^{-1 / 2}$.

We shall now prove a crucial lemma about $X$. It is an easy consequence of Lemmas 7 and 9.

Lemma 10. Let $N \in L$, let $n \in[\log N$, $\exp N]$, let $\epsilon>0$, and let $x_{1}, \ldots, x_{n}$ be a R.I.S. with constant $1+\epsilon$. Then $\left\|\sum_{i=1}^{n} x_{i}\right\| \leqslant\left(1+\epsilon+\epsilon^{\prime}\right) n f(n)^{-1}$.

Proof. It is obvious from the implicit definition of the norm in $X$ that it satisfies a lower $f$-estimate. Let $g$ be the function defined before the last lemma in the case $K_{0}=K$. As usual let $x=\sum_{i=1}^{n} x_{i}$. Since $g \leqslant \phi$, it is clear that every vector in $X$ is either normed by an $(M, g)$-form or has the supremum 
norm. It is also clear that the second possibility does not happen in the case of vectors of the form $E x$ when $\lambda(E) \geqslant 1$. Since $g \in \mathscr{F}$ and, as we commented above, $g \geqslant f^{1 / 2}$, all the hypotheses of Lemma 7 are satisfied. It follows that $\left\|\sum_{i=1}^{n} x_{i}\right\| \leqslant\left(1+\epsilon+\epsilon^{\prime}\right) G(n)$. By Lemma 9, $G(n)=n f(n)^{-1}$, so the lemma is proved.

Lemma 11. Let $N \in L$, let $0<\epsilon<1 / 4$, let $M=N^{\epsilon}$, and let $x_{1}, \ldots, x_{N}$ be a R.I.S. with constant $1+\epsilon$. Then $\sum_{i=1}^{N} x_{i}$ is an $\ell_{1+}^{M}$-vector with constant $(1+4 \epsilon)$.

Proof. Let $m=N / M$, let $x=\sum_{i=1}^{N} x_{i}$, and for $1 \leqslant j \leqslant M$ let $y_{j}=$ $\sum_{i=(j-1) m+1}^{j m} x_{i}$. Then each $y_{j}$ is the sum of a R.I.S. of length $m$ with constant $(1+\epsilon)$. By Lemma 10 we have $\left\|y_{j}\right\| \leqslant(1+2 \epsilon) m f(m)^{-1}$ for every $j$ while $\left\|\sum_{j=1}^{m} y_{j}\right\|=\|x\| \geqslant N f(N)^{-1}$. It follows that $x$ is an $\ell_{1+}^{M}$-vector with constant at most $(1+2 \epsilon) f(N) / f(m)$. But $m=N^{1-\epsilon}$, so $f(N) / f(m) \leqslant(1-\epsilon)^{-1}$. The result follows.

The next lemma is similar to Lemma 10 but is more complicated.

Lemma 12. Let $k \in K$ and $x_{1}^{*}, \ldots, x_{k}^{*}$ be a special sequence of length $k$, where each $x_{i}^{*}$ is an $\left(M_{i}, f\right)$-form. Let $x_{1}, \ldots, x_{k}$ be a sequence of successive vectors such that every $x_{i}$ is a normalized R.I.S. vector of length $M_{i}$ and constant $1+\epsilon / 4, \epsilon=1 / 10$. Assume that $\left|\left(\sum_{i=1}^{k} x_{i}^{*}\right)\left(\sum_{i=1}^{k} E x_{i}\right)\right| \leqslant 2$ for every interval E. Then

$$
\left\|\sum_{i=1}^{k} x_{i}\right\| \leqslant(1+2 \epsilon) k / f(k) .
$$

Proof. We know by Lemma 11 that each $x_{i}$ is an $\ell_{1+}^{N_{i}}$-average with constant $1+\epsilon$, where $N_{i}=M_{i}^{\epsilon / 4}$. Also $M_{1}=j_{2 k}$ and $M_{1}^{\epsilon / 4}=N_{1} \geqslant 4 M_{f}(k / \epsilon) / \epsilon f^{\prime}(1)$. Recall that $\sigma$ was chosen so that, if $z_{1}, \ldots, z_{s}$ is a sequence of successive vectors in $\mathbf{Q}, S=\sigma\left(z_{1}, \ldots, z_{s}\right)$, and $z=\sum_{i=1}^{s} z_{i}$, then $(1 / 20) f\left(S^{1 / 40}\right)^{1 / 2} \geqslant$ $|\operatorname{supp}(z)|$. This and the lower bound for $N_{1}$ ensure that $x_{1}, \ldots, x_{k}$ is a R.I.S. of length $k$ with constant $1+\epsilon$.

To prove Lemma 12 we shall apply Lemma 7. First, we shall show that, if $z_{1}^{*}, \ldots, z_{k}^{*}$ is any special sequence of functionals of length $k$ and $E$ is any interval, then $\left|z^{*}(E x)\right| \leqslant 1 / 4$, where $z^{*}$ is the $(k, \sqrt{f})$-form $f(k)^{-1 / 2} \sum_{i=1}^{k} z_{i}^{*}$ and $x=\sum_{i=1}^{k} x_{i}$.

Indeed, let $t$ be maximal such that $z_{t}^{*}=x_{t}^{*}$ or zero if no such $t$ exists. Suppose $i \neq j$ or one of $i, j$ is greater than $t+1$. Then, since $\sigma$ is an injection, we can find $L_{1} \neq L_{2} \in L$ such that $z_{i}^{*}$ is an $\left(L_{1}, f\right)$-form and $x_{j}$ is the normalized sum of a R.I.S. of length $L_{2}$ and also an $\ell_{1+}^{L_{2}^{\prime}}$-average with constant $1+\epsilon$, where $L_{2}^{\prime}=L_{2}^{\epsilon / 4}$. Just as at the end of $\S 2$, we can now use Lemmas 4 and 5 to show that $\left|z_{i}^{*}\left(E x_{j}\right)\right|<k^{-2}$. If $L_{1}<L_{2}$, it follows from the definition of $L$ that $L_{1}<L_{2}^{\prime}$. We know that $L_{1} \geqslant j_{2 k}$ since $L_{1}$ appears in a special sequence 
of length $k$. Lemma 4 thus gives $\left|z_{i}^{*}\left(E x_{j}\right)\right|=\left|\left(E z_{i}^{*}\right)\left(x_{j}\right)\right| \leqslant 3(1+\epsilon) / f\left(L_{1}\right)$. The conclusion in this case now follows from the fact that $f(l) \geqslant 4 k^{2}$ when $l \geqslant j_{2 k}$.

If $L_{2}<L_{1}$, we apply Lemma 5 with $\epsilon_{1}=1$ to the nonnormalized sum $x_{j}^{\prime}$ of the R.I.S., the normalized sum of which is $x_{j}$. The definition of $L$ gives us that $M_{f}\left(L_{2}\right)<L_{1}$, so Lemma 5 gives $\left|z_{i}^{*}\left(E x_{j}^{\prime}\right)\right| \leqslant 3$. It follows from the lower $f$-estimate that $\left\|x_{j}^{\prime}\right\| \geqslant L_{2} / f\left(L_{2}\right)$. The conclusion now follows because $l \geqslant j_{2 k}$ implies that $f(l) / l \leqslant 1 / 4 k^{2}$.

Now choose an interval $F$ such that

$$
\left|\left(\sum_{i=1}^{t} z_{i}^{*}\right)(E x)\right|=\left|\left(\sum_{i=1}^{k} x_{i}^{*}\right)(F x)\right| \leqslant 2 .
$$

It follows that

$$
\left|\left(\sum_{i=1}^{k} z_{i}^{*}\right)(E x)\right| \leqslant 2+\left|z_{t+1}^{*}\left(x_{t+1}\right)\right|+k^{2} \cdot k^{-2} \leqslant 4 .
$$

We finally obtain that $\left|z^{*}(E x)\right| \leqslant 4 f(k)^{-1 / 2}<1 / 4$ as claimed.

Now let $\phi^{\prime}$ be the function

$$
\phi^{\prime}(x)= \begin{cases}\left(\log _{2}(x+1)\right)^{1 / 2} & \text { if } x \in K, x \neq k, \\ \log _{2}(x+1) & \text { otherwise. }\end{cases}
$$

Let $g^{\prime}$ be the function obtained from $\phi^{\prime}$ by the construction explained before Lemma 9 , in the case $K_{0}=K \backslash\{k\}$. Lemma 9 in this case tells us that $g^{\prime}(l)=f(l)$ for every $l \in L \cup\{k\}$.

It follows from what we have just shown about special sequences of length $k$ that

$$
1 / 4<\|E x\| \leqslant \sup \left\{\left|x^{*}(E x)\right|: M \geqslant 2, x^{*} \text { is an }\left(M, g^{\prime}\right) \text {-form }\right\}
$$

whenever $E$ is an interval of length at least 1. Since $x$ is the sum of a R.I.S., Lemma 7 implies that $\|x\| \leqslant(1+2 \epsilon) k^{\prime}(k)^{-1}=(1+2 \epsilon) k / f(k)$.

We shall now prove that $X$ is H.I. As we noted earlier, this implies that $X$ contains no unconditional basic sequence, but in proving that $X$ is H.I. we shall more or less have proved that directly anyway.

Let $Y, Z$ be two infinite-dimensional subspaces of $X$ such that $Y \cap Z=$ $\{0\}$. Our aim is now to show that the projection from $Y+Z$ to $Y$ given by $y+z \mapsto y$ is not continuous. To do this, we shall construct, for every $\delta>0$, vectors $y \in Y$ and $z \in Z$ such that $\delta\|y+z\|>\|y-z\|$. This implies that the above projection has norm at least $(1-\delta) \delta^{-1} / 2$, proving the result. So let us now choose $\delta>0$, and let $k \in K$ be an integer such that $f(k)^{-1 / 2}<\delta / 4$.

By standard arguments, we may assume that both $Y$ and $Z$ are spanned by block bases. Since $X$ satisfies a lower $f$-estimate, Lemma 3 tells us that every block subspace of $X$ contains, for every $\epsilon>0$ and $N \in \mathbb{N}$, an $\ell_{1+}^{N}$-average 
with constant $1+\epsilon$. It is also immediate from the definition of the norm that every vector either has the supremum norm or satisfies the inequality

$$
\|E x\| \leqslant \sup \left\{\left|x^{*}(E x)\right|: M \geqslant 2, x^{*} \text { is an }(M, g) \text {-form }\right\},
$$

where $g$ is the function obtained from $\phi$ after the proof of Lemma 8 in the case $K_{0}=K$. This allows us to make the following construction.

Let $x_{1} \in Y$ be a normalized R.I.S. vector of length $M_{1}=j_{2 k} \in L$ and constant $(1+\epsilon / 4)$, where $\epsilon=1 / 10$ and $M_{1}^{\epsilon / 4} \geqslant N_{1}=4 M_{f}(k / \epsilon) / \epsilon f^{\prime}(1)$.

Let the nonnormalized R.I.S. whose sum is $x_{1}$ be $x_{11}, \ldots, x_{1 M_{1}}$. By Lemma $11, x_{1}$ is an $\ell_{1+}^{N_{1}}$-average with constant $1+\epsilon$. By Lemma 7 , we have $\left\|x_{1}\right\| \leqslant(1+\epsilon) M_{1} g\left(M_{1}\right)^{-1}\left\|x_{11}\right\|$. For each $j$ between 1 and $M_{1}$ let $x_{1 j}^{*}$ be a support functional for $x_{1 j}$ and let $x_{1}^{\prime *}$ be the $\left(M_{1}, g\right)$-form $g\left(M_{1}\right)^{-1} \sum_{j=1}^{M_{1}} x_{1 j}^{*}$. Then $x_{1}^{\prime *}\left(x_{1}\right) \geqslant(1+\epsilon)^{-1}\left\|x_{1}\right\|$. By continuity and the density of $\mathbf{Q}$ it follows that there exists an $\left(M_{1}, g\right)$-form $x_{1}^{*} \in \mathbf{Q}$ such that $\left|x_{1}^{*}\left(x_{1}\right)-1 / 2\right| \leqslant k^{-1}$ and $\operatorname{ran}\left(x_{1}^{*}\right)=\operatorname{ran}\left(x_{1}\right)$. Also, note that by Lemma 9 there is no difference between an $\left(M_{1}, g\right)$-form and an $\left(M_{1}, f\right)$-form.

Now let $M_{2}=\sigma\left(x_{1}^{*}\right)$, and pick a normalized R.I.S. vector $x_{2} \in Z$ of length $M_{2}$ with constant $1+\epsilon / 4$ such that $x_{1}<x_{2}$. Then $x_{2}$ is an $\ell_{1+}^{N_{2}}$-average with constant $1+\epsilon$, where $N_{2}=M_{2}^{\epsilon / 4}$. As above, we can find an $\left(M_{2}, g\right)$-form $x_{2}^{*}$ such that $\left|x_{2}^{*}\left(x_{2}\right)-1 / 2\right| \leqslant k^{-1}$ and $\operatorname{ran}\left(x_{2}^{*}\right)=\operatorname{ran}\left(x_{2}\right)$.

Continuing in this manner, we obtain a pair of sequences $x_{1}, \ldots, x_{k}$ and $x_{1}^{*}, \ldots, x_{k}^{*}$ with various properties we shall need. First, $x_{i} \in Y$ when $i$ is odd and $Z$ when $i$ is even. Second, $\left\|x_{i}\right\|=1$ for every $i$ and $\left\|x_{i}^{*}\right\| \leqslant 1$. We also know that $\left|x_{i}^{*}\left(x_{i}\right)-1 / 2\right| \leqslant 1 / k$ for each $i$. As in the proof of Lemma 12, our choice of $\sigma$ and the lower bound for $N_{1}$ ensure that $x_{1}, \ldots, x_{k}$ is a R.I.S. of length $k$. Finally, and perhaps most importantly, the sequence $x_{1}^{*}, \ldots, x_{k}^{*}$ has been carefully chosen to be a special sequence of length $k$. It follows immediately from the implicit definition of the norm and the fact that $\operatorname{ran}\left(x_{i}^{*}\right) \subset \operatorname{ran}\left(x_{i}\right)$ for each $i$ that

$$
\left\|\sum_{i=1}^{k} x_{i}\right\| \geqslant f(k)^{-1 / 2} \sum_{i=1}^{k} x_{i}^{*}\left(x_{i}\right) \geqslant f(k)^{-1 / 2}(k / 2-1) .
$$

The proof will be complete if we can find a suitable upper bound for $\left\|\sum_{i=1}^{k}(-1)^{i-1} x_{i}\right\|$. For this we apply Lemma 12 . We need to show that

$$
\left|\left(\sum_{i=1}^{k} x_{i}^{*}\right)\left(\sum_{i=1}^{k}(-1)^{i-1} E x_{i}\right)\right| \leqslant 2
$$

for every interval $E$. This follows easily from the fact that $x_{i}^{*}\left(x_{i}\right)$ is almost exactly $1 / 2$ for every $i$. Lemma 12 therefore shows that $\left\|\sum_{i=1}^{k}(-1)^{i-1} x_{i}\right\| \leqslant$ $(1+2 \epsilon) k f(k)^{-1}$.

We have now constructed two vectors $y \in Y$, the sum of the odd-numbered $x_{i}$ 's, and $z \in Z$, the sum of the even-numbered $x_{i}$ 's, such that $\|y+z\| \geqslant$ 
$(1 / 3) f(k)^{1 / 2}\|y-z\| \geq \delta^{-1}\|y-z\|$. Hence, $Y$ and $Z$ do not form a topological direct sum, so $X$ is H.I. If $X$ contained an unconditional basic sequence $x_{1}, x_{2}, \ldots$ then the subspace generated by this sequence would split into a direct sum of the subspaces generated by $\left\{x_{2 n-1}: n \in \mathbb{N}\right\}$ and $\left\{x_{2 n}: n \in \mathbb{N}\right\}$. It follows that $X$ does not contain an unconditional basic sequence. The reader will observe that it is easy to use the preceding argument to show this directly. In the next section, we shall examine some of the other consequences of a space being H.I., but first we shall observe that $X$ is reflexive. Recall that a basis $\left(x_{n}\right)_{n=1}^{\infty}$ of a Banach space is shrinking if for every continuous linear functional $x^{*}$ and every $\epsilon>0$ there exists $n \in \mathbb{N}$ such that the norm of $x^{*}$ restricted to the span of $x_{n}, x_{n+1}, \ldots$ is at most $\epsilon$. It is boundedly complete if, given any sequence of scalars $\left(a_{n}\right)_{n=1}^{\infty}$ for which the partial sums $\sum_{n=1}^{N} a_{n} x_{n}$ are bounded, the sum $\sum_{n=1}^{\infty} a_{n} x_{n}$ converges. It is a well-known result of James (see, e.g., [LT, §1.b]) that a Banach space with a shrinking and boundedly complete basis is reflexive.

It follows immediately from the fact that $X$ satisfies a lower $f$-estimate that the standard basis $\mathbf{e}_{1}, \mathbf{e}_{2}, \ldots$ is boundedly complete. Now suppose that it is not a shrinking basis. Then we can find $\epsilon>0$, a norm-1 functional $x^{*} \in X^{*}$, and a sequence of successive normalized blocks $x_{1}, x_{2}, \ldots$ such that $x^{*}\left(x_{n}\right) \geqslant \epsilon$ for every $n$. It follows that $\sum_{n \in A} x_{n}$ is an $\ell_{1+}^{|A|}$-vector with constant $\epsilon^{-1}$ for every $A \subset \mathbb{N}$. Given $N \in L$ we may construct a R.I.S. $y_{1}, \ldots, y_{N}$ with constant $\epsilon^{-1}$ where $y_{i}$ is of the form $\lambda_{i} \sum_{j \in A_{i}} x_{j}$, with $\lambda_{i} \geqslant\left|A_{i}\right|^{-1}$. Then $x^{*}\left(y_{1}+\cdots+y_{N}\right) \geqslant \epsilon N$. For $N$ sufficiently large, this contradicts Lemma 10.

\section{OPERATORS ON H.I. SPACES}

In this section, we shall prove some results about H.I. spaces over $\mathbb{C}$. This is because we shall need to use a little spectral theory. In the next section we shall show that some of the results carry over to the real case. We do not know of a direct proof. Later, however, we will give a proof for our specific example (over the reals) which does not mention complex numbers. The elementary background in spectral theory that we use can be found in [DS, Part I, Chapter VII], or in many other textbooks.

Let $X$ be a complex Banach space, and let $T$ be a bounded linear operator from $X$ into itself. We say that $\lambda \in \mathbb{C}$ is infinitely singular for $T$ if, for every $\epsilon>0$, there exists an infinite-dimensional subspace $Y_{\epsilon}$ of $X$ such that the restriction of $T-\lambda I$ to $Y_{\epsilon}$ has norm at most $\epsilon$.

Saying that $\lambda$ is not infinitely singular for $T$ is equivalent to saying that $T-\lambda I$ is an isomorphism on some finite-codimensional subspace of $X$. Since this property is clearly unaffected by a small enough perturbation, it follows that

$$
F_{T}=\{\lambda \in \mathbb{C}: \lambda \text { not infinitely singular for } T\}
$$

is an open subset of $\mathbb{C}$. Notice that $\operatorname{ker}(T-\lambda I)$ is finite dimensional when $\lambda \in F_{T}$. We shall now prove some lemmas about $F_{T}$. These are basically well-known facts in operator theory. 
Lemma 13. If $\lambda \in F_{T}$ and if $\left(x_{n}\right)$ is a bounded sequence such that $(T-\lambda I) x_{n}$ is norm-convergent, then $\left(x_{n}\right)$ has a norm-convergent subsequence; furthermore, the image by $T-\lambda I$ of any closed subspace of $X$ is closed.

Proof. Let $S=T-\lambda I$, let $Y$ be a finite-codimensional subspace on which $S$ is an isomorphism, and let $X=Y \oplus Z$. Let $x_{n}=y_{n}+z_{n}$ with $y_{n} \in Y$ and $z_{n} \in Z$. Then $S x_{n}=S y_{n}+S z_{n}$. Since $Z$ is finite dimensional and $\left(x_{n}\right)$ is bounded, we can pass to a subsequence such that $S z_{n}$ converges. Since $S x_{n}$ converges, this gives us that $S y_{n}$ converges (relabeling the subsequence as $\left.S y_{n}\right)$. Since $S$ is an isomorphism on $Y$, it follows that $y_{n}$ converges. Finally pass to a further subsequence on which $z_{n}$ converges. To prove the second assertion, note that if $F$ is a closed subspace of $X$, then $F=F \cap Y+G$, for some finite-dimensional $G$, and hence $T(F)=T(F \cap Y)+T(G)$ is closed.

Lemma 14. If $\lambda \in \partial \operatorname{Sp}(T) \cap F_{T}$, then $\lambda$ is an eigenvalue of $T$ with finite multiplicity.

Proof. Since $\lambda \in \partial S p(T)$ it is an approximate eigenvalue of $T$. In other words, there exists a sequence $\left(x_{n}\right)$ of norm- 1 vectors with $T x_{i}-\lambda x_{i} \rightarrow 0$. By the previous lemma $\left(x_{n}\right)$ has a convergent subsequence. But then the limit of the subsequence is an eigenvector with eigenvalue $\lambda$.

The next lemma follows easily from well known facts in Fredholm theory. The argument here is elementary. It was shown to us by W. B. Johnson, as was the proof of Lemma 16.

Lemma 15. If $\lambda \in \partial S p(T) \cap F_{T}$ then $\lambda$ is an isolated point of $S p(T)$.

Proof. Since $F_{T}$ is open, it is enough to show that $\lambda$ is an isolated point of $\partial S p(T) \cap F_{T}$. Suppose that this is not the case. Then there exists a sequence $\left(\lambda_{n}\right)$ in $\partial S p(T) \cap F_{T}$ converging to $\lambda$, with $\lambda_{n} \neq \lambda$ for every $n$. Since $\lambda_{n} \in F_{T}, \lambda_{n}$ is an eigenvalue, by Lemma 14. Let $x_{n}$ be a norm-1 eigenvector with eigenvalue $\lambda_{n}$. By Lemma 13, since $(T-\lambda I) x_{n}$ tends to 0 , we may assume that $\left(x_{n}\right)$ is norm-convergent to some (norm-1) vector $x$ such that $T x=\lambda x$. Let $Y$ be the closed subspace of $X$ generated by the sequence $\left(x_{n}\right)$. Let $U$ be the restriction of $T-\lambda I$ to $Y$. It is clear that $Y$ is invariant under $U$ and that $U Y$ is dense in $Y$. Furthermore, since $(T-\lambda I) Y=U Y$ and $\lambda \in F_{T}$, it follows from Lemma 13 that $U Y$ is closed and, hence, that $U Y=Y$. Since $x \in Y$, we know that $Y_{0}=\operatorname{ker} U$ is not $\{0\}$ and that it is finite dimensional. We can therefore write $Y$ as a direct sum $Y_{0}+Y_{1}$. We have that $U Y_{1}=Y$, so for small $\epsilon$ it is still true that $(U-\epsilon I) Y_{1}=Y$. But since $(U-\epsilon I) Y_{0}=Y_{0}$ when $\epsilon \neq 0$, this yields that $\operatorname{ker}(U-\epsilon I) \neq\{0\}$, for every small $\epsilon$, contradicting the fact that $\lambda \in \partial S p(T)$.

Lemma 16. Let $S$ be a bounded linear operator from $X$ to itself. Suppose that $S p(S)=\{0\}$. If $X$ is infinite dimensional, then 0 is infinitely singular for $S$.

Proof. Suppose that $0 \in F_{S}$ but that $X$ is infinite dimensional. Then $S$ is an isomorphism on some finite-codimensional subspace $Z$ of $X$. Replacing $S$ by an appropriate multiple, we may assume that $\|S z\| \geqslant\|z\|$ for every $z \in Z$. Define $Z_{0}=Z, Z_{1}=Z \cap S Z, \ldots, Z_{k+1}=Z \cap S Z_{k}$. All these subspaces of $X$ are infinite dimensional. If $z$ is a nonzero element of $Z_{k}$, then $z=S^{k} z_{0}$ 
for some $z_{0} \in Z$ and $0<\left\|z_{0}\right\| \leqslant\left\|S^{k} z_{0}\right\|$. This shows that $\left\|S^{k}\right\| \geqslant 1$ for every $k$, contradicting the fact that the spectral radius of $S$ is 0 .

Lemma 17. If $X$ is infinite dimensional then $F_{T} \neq \mathbb{C}$.

Proof. Assume that $F_{T}=\mathbb{C}$. It follows from Lemma 15 that every point in $\partial S p(T)$ is isolated in $S p(T)$, so the spectrum of $T$ is finite. Let us write $S p(T)=\left\{\lambda_{1}, \ldots, \lambda_{n}\right\}$. Consider the polynomial $P=\prod_{i=1}^{n}\left(z-\lambda_{i}\right)$. For every $\lambda \neq 0$, we can write $P-\lambda=\prod_{i=1}^{n}\left(z-\mu_{i}\right)$, where $\mu_{i} \notin S p(T)$ for every $i=1, \ldots, n$. It follows that $(P-\lambda)(T)=P(T)-\lambda I$ is invertible for every $\lambda \neq 0$ and, therefore, that $S p(P(T))=\{0\}$. Since $X$ is infinite dimensional, Lemma 16 tells us that 0 is infinitely singular for $P(T)$. Hence, there exists a normalized basic sequence $\left(x_{n}\right)$ such that $P(T) x_{n} \rightarrow 0$. Writing $P(T)=\left(T-\lambda_{1} I\right) P_{1}(T)$ and making repeated applications of Lemma 13, we arrive at the absurd conclusion that some subsequence of $\left(x_{n}\right)$ is convergent.

Suppose now that $X$ is a complex H.I. Banach space. Let $T$ be a bounded linear operator from $X$ into itself. It follows easily from the H.I. property that there exists at most one value $\lambda_{0}$ that is infinitely singular for $T$. If $\lambda_{0}$ is infinitely singular for $T$, the H.I. property implies that $T-\lambda_{0} I$ is not an isomorphism on any infinite-dimensional subspace of $X$. In other words, $T-\lambda_{0} I$ is strictly singular.

We have therefore proved the following theorem.

Theorem 18. If $X$ is a complex H.I. Banach space then every bounded linear operator $T$ from $X$ into $X$ can be written $T=\lambda I+S$, where $\lambda \in \mathbb{C}$ and $S$ is strictly singular. The spectrum of $T$ is finite or consists of $\lambda$ and a sequence $\left(\lambda_{n}\right)$ of eigenvalues with finite multiplicity converging to $\lambda$.

Corollary 19. A complex H.I. space $X$ is not isomorphic to any proper subspace and, in particular, is not isomorphic to its hyperplanes.

\section{FURTHER PROPERTIES}

We shall now show how to pass from the complex case back to the real case. The following lemma will be useful; it was shown to us by R. G. Haydon. As before, it implies that there is no isomorphism between $X$ and a proper subspace.

Lemma 20. Suppose $X$ is a real H.I. space and $T$ a bounded linear operator from $X$ into itself. If we denote by $S$ the natural extension of $T$ to the complexification of $X$ then the spectrum of $S$ is invariant by conjugation and the part in the upper complex plane is finite or consists of a converging sequence.

Proof. If $\lambda \notin F_{S}$ is real, there exists for every $\epsilon>0$ a (real) infinite-dimensional subspace $Y_{\epsilon}$ of $X$ such that $\left\|T-\lambda \operatorname{Id}_{Y_{\epsilon}}\right\|<\epsilon$ on $Y_{\epsilon}$. Since $X$ is H.I., it follows that $\mathbb{C} \backslash F_{S}$ contains at most one real element. Let now $\lambda, \mu \notin F_{S}$, and $\mu \notin\{\lambda, \bar{\lambda}\}$. We may assume that $\lambda$ is not real. Let

$$
T_{\lambda}=T^{2}-2 \operatorname{Re} \lambda T+|\lambda|^{2} \mathrm{Id}
$$

Then $(S-\bar{\lambda} \operatorname{Id})(S-\lambda \mathrm{Id})(x+i y)=T_{\lambda} x+i T_{\lambda} y$. For every $\epsilon>0$ it is thus possible to find an infinite-dimensional subspace $Y_{\epsilon}$ of $X$ such that $\left\|T_{\lambda}\right\|<\epsilon$ 
on $Y_{\epsilon}$. Since $X$ is H.I., we may assume the same for $T_{\mu}$ on the same $Y_{\epsilon}$. Now, $T_{\lambda}-T_{\mu}=a T+b$ Id for some $a, b \in \mathbb{R}$, not both 0 , and it has norm less than $2 \epsilon$ on $Y_{\epsilon}$. Thus $a \neq 0$. We obtain that $T$ is nearly equal to $(-b / a)$ Id on $Y_{\epsilon}$. Since $T_{\lambda}$ is nearly 0 on $Y_{\epsilon}$, we get easily that $-b / a$ must be a root of the polynomial $(z-\bar{\lambda})(z-\lambda)$, which of course is impossible.

We know, therefore, that $\mathbb{C} \backslash F_{S}$ contains at most a pair $(\lambda, \bar{\lambda})$, and the rest of the proof is as in $\S 4$.

We have therefore proved the following result.

Theorem 21. If $X$ is a real H.I. space (e.g., $X$ could be the real version of the space from $\S 3$ ) then $X$ is not isomorphic to any proper subspace. In particular, $X$ is not isomorphic to its hyperplanes.

As we mentioned earlier, we have a direct proof of this result (i.e., one that does not mention the complex numbers) when $X$ is the real version of the space from $\S 3$. It uses a lemma which is of independent interest and has other applications [G2].

Suppose then that $X$ is indeed the Banach space over $\mathbb{R}$ that was constructed in $\S 3$.

Lemma 22. Let $Y$ be a block subspace of $X$, and let $T$ be a bounded linear operator from $Y$ to $X$. There exists $\lambda \in \mathbb{R}$ such that

$$
T\left(x_{n}\right)-\lambda x_{n} \rightarrow_{n} 0
$$

for every sequence $\left(x_{n}\right)$ such that $x_{n} \in Y$ is an $\ell_{1+}^{n}$-average with constant $1+$ $\epsilon / 4, \epsilon=1 / 10$ for every integer $n$.

We shall prove first a preliminary result:

Lemma 23. Let $Y$ be a block subspace of $X$, and let $\left(y_{n}\right)$ be a sequence in $Y$ such that $y_{n}$ is an $\ell_{1+}^{n}$-average with constant $1+\epsilon / 4, \varepsilon=1 / 10$ for every $n$. For every bounded linear operator $T$ from $Y$ to $X$, we have

$$
d\left(T y_{n}, \mathbb{R} y_{n}\right) \rightarrow_{n} 0
$$

Proof. Let $\left(f_{n}\right)$ be the basis of $Y$. Since it converges weakly to 0 , we may perturb $T$ slightly in such a way that, for every $n, T\left(f_{n}\right)$ is a finite block with respect to the basis of $X$ and so that $\min \operatorname{supp}\left(T\left(f_{n}\right)\right) \rightarrow_{n} \infty$.

For $y \in Y$ with finite support, let $I(y)$ be the smallest interval containing the supports of $y$ and $T(y)$. Since the $\left(y_{n}\right)$ have increasing lengths, we may assume passing to a subsequence and after a small perturbation that they are successive. We can then assume also that $I\left(y_{n}\right)<I\left(y_{n+1}\right)$.

If the result is not true, we may assume, on passing to a subsequence, that there exists $\delta>0$ such that $d\left(T y_{n}, \mathbb{R} y_{n}\right)>\delta$ for every $n$. By the HahnBanach theorem, we can find $y_{n}^{*} \in B\left(X^{*}\right)$, for each $n$, such that $y_{n}^{*}\left(T y_{n}\right)>\delta$ and $y_{n}^{*}\left(y_{n}\right)=0$. We may also assume that $\operatorname{ran}\left(y_{n}^{*}\right) \subset I\left(y_{n}\right)$.

We now claim that, for every $p \in L$ and $m \in \mathbb{N}$, there exist a normalized R.I.S. vector $x \in Y$ of length $p$ and constant $1+\epsilon / 4$ and a $(p, f)$-form $x^{*}$ such that $m<I(x), \operatorname{ran}\left(x^{*}\right) \subset I(x)$, and

$$
x^{*}(x)=0, \quad x^{*}(T(x))>\delta / 2 .
$$


Indeed, it is clear that we can select $y_{n_{1}}, \ldots, y_{n_{p}}$ forming a R.I.S. of length $p \in L$ and constant $1+\epsilon / 4$ such that $m<I\left(y_{n_{1}}\right)^{p}$. By Lemma 10, we know that

$$
\left\|\sum_{i=1}^{p} y_{n_{i}}\right\| \leqslant 2 p / f(p) .
$$

Letting $x^{*}=\left(y_{n_{1}}^{*}+\cdots+y_{n_{p}}^{*}\right) / f(p) \in B\left(X^{*}\right)$, we obtain $x^{*}\left(\sum_{i=1}^{p} T\left(y_{n_{i}}\right)\right)>$ $\delta p / f(p)$. On the other hand, $x^{*}\left(\sum_{i=1}^{p} y_{n_{i}}\right)=0$. We simply have to choose $x$ to be a norm one multiple of $y_{n_{1}}+\cdots+y_{n_{p}}$.

The rest of the proof of the lemma is similar to the proof that $X$ is H.I. at the end of $\S 3$. Let $x_{1} \in Y$ be a normalized R.I.S. vector of length $M_{1}=j_{2 k} \in L$ and constant $(1+\epsilon / 4)$, where $M_{1}^{\epsilon / 4}=N_{1} \geqslant 4 M_{f}(k / \epsilon) / \epsilon f^{\prime}(1)$, and let $x_{1}^{*}$ be an $\left(M_{1}, f\right)$-form in $\mathbf{Q}$ such that $\left|x_{1}^{*}\left(x_{1}\right)\right| \leqslant k^{-2}$ and $x_{1}^{*}\left(T\left(x_{1}\right)\right)>\delta / 2$. We assume, as we may, that $\operatorname{ran}\left(x_{1}^{*}\right) \subset I\left(x_{1}\right)$.

Now let $M_{2}=\sigma\left(x_{1}^{*}\right)$, and pick a normalized R.I.S. vector $x_{2} \in Y$ of length $M_{2}$ with constant $1+\epsilon / 4$ such that $I\left(x_{1}\right)<I\left(x_{2}\right)$ and an $\left(M_{2}, f\right)$-form $x_{2}^{*} \in \mathbf{Q}$ such that $\operatorname{ran}\left(x_{2}^{*}\right) \subset I\left(x_{2}\right),\left|x_{2}^{*}\left(x_{2}\right)\right| \leqslant k^{-2}$ and $x_{2}^{*}\left(T\left(x_{2}\right)\right)>\delta / 2$.

Continuing in this manner, we obtain a pair of sequences $x_{1}, \ldots, x_{k}$ and $x_{1}^{*}, \ldots, x_{k}^{*}$ with various properties we need. First, the intervals $I\left(x_{i}\right)$ are successive, and we have $\operatorname{ran}\left(x_{i}^{*}\right) \subset I\left(x_{i}\right),\left\|x_{i}\right\|=1$, and $\left\|x_{i}^{*}\right\| \leqslant 1$ for every $i$. We also know that $\left|x_{i}^{*}\left(x_{i}\right)\right| \leqslant k^{-2}$ and $x_{i}^{*}\left(T\left(x_{i}\right)\right)>\delta / 2$ for each $i$. It is easy to show that $\left|\left(\sum_{i=1}^{k} x_{i}^{*}\right)\left(\sum_{i=1}^{k} E x_{i}\right)\right| \leqslant 2$ for every interval $E$, so we can apply Lemma 12 to obtain $\left\|\sum_{i=1}^{k} x_{i}\right\| \leqslant 2 k / f(k)$.

Finally, as with the earlier argument, the sequence $x_{1}^{*}, \ldots, x_{k}^{*}$ has been chosen to be a special sequence of length $k$. It follows from the definition of the norm and the fact that $I\left(x_{i}\right)<I\left(x_{i+1}\right), \operatorname{ran}\left(x_{i}^{*}\right) \subset I\left(x_{i}\right)$ for each $i$ that

$$
\left\|\sum_{i=1}^{k} T\left(x_{i}\right)\right\|>\delta k f(k)^{-1 / 2} / 2 .
$$

From this and the preceding estimate, we can deduce that $\|T\| \geqslant \delta \sqrt{f(k)} / 4$, for every $k \in K$, contradicting the boundedness of $T$.

Proof of Lemma 22. We know by Lemma 23 that, for every sequence $\left(x_{n}\right)$ of $\ell_{1+}^{n}$-averages in $Y$ with constant $1+\epsilon / 4$, there exists $\lambda \in \mathbb{R}$ and a subsequence $\left(x_{n}^{\prime}\right)$ such that $T\left(x_{n}^{\prime}\right)-\lambda x_{n}^{\prime} \rightarrow 0$. It is easy to deduce from this that $T\left(x_{n}\right)-$ $\lambda x_{n} \rightarrow 0$, by mixing subsequences with possibly different values of $\lambda$. One can deduce that $\lambda$ is independent of the sequence $\left(x_{n}\right)$ by the same argument.

It is clear now that $U=T-\lambda I$ is strictly singular, where $I$ denotes the injection from $Y$ to $X$. Indeed, every infinite-dimensional subspace of $Y$ contains sequences of $\ell_{1+}^{n}$-averages $\left(x_{n}\right)$ with constant $1+\epsilon / 4$, for which $U\left(x_{n}\right) \rightarrow 0$.

It is easy to finish the proof of the hyperplane property. Let $T$ be any bounded linear operator from $X$ to $X$. We know that, for some $\lambda \in \mathbb{R}$, $S=T-\lambda$ Id is strictly singular. If $\lambda=0, T$ cannot be an isomorphism from 
$X$ into $X$. If $\lambda \neq 0$, it is known that $\lambda \mathrm{Id}+S$ is a Fredholm operator with index 0 , so $T$ cannot be an isomorphism from $X$ onto a proper subspace.

\title{
REFERENCES
}

[BP] C. Bessaga and A. Petczyński, A generalization of results of $R$. C. James concerning absolute bases in Banach spaces, Studia Math. 17 (1958), 165-174.

[CS] P. G. Casazza and T. Shura, Tsirelson's space, Lecture Notes in Math., vol. 1363, Springer Verlag, New York, 1988.

[DS] N. Dunford and J. Schwartz, Linear operators, Vol. I, Interscience, New York, 1958.

[E] P. Enflo, $A$ counterexample to the approximation property in Banach spaces, Acta Math. 130 (1973), 309-317.

[G1] W. T. Gowers, A solution to Banach's hyperplane problem, preprint.

[G2] _ A solution to the Schroeder-Bernstein problem for Banach spaces, preprint.

[L] J. Lindenstrauss, Some aspects of the theory of Banach spaces, Adv. Math. 5 (1970), 159180.

[LP] J. Lindenstrauss and A. Petczyński, Contributions to the theory of classical Banach spaces, J. Funct. Anal. 8 (1971), 225-249.

[LT] J. Lindenstrauss and L. Tzafriri, Classical Banach spaces, Vol. I, Springer-Verlag, New York, 1977.

[MR] B. Maurey and H. P. Rosenthal, Normalized weakly null sequences with no unconditional subsequence, Studia Math. 61 (1977), 77-98.

[OS] E. Odell and T. Schlumprecht, The distortion problem, preprint.

[S1] T. Schlumprecht, An arbitrarily distortable Banach space, Israel J. Math. 76 (1991), 81-95.

[S2] _ A complementably minimal Banach space not containing $c_{0}$ or $\ell_{p}$, preprint.

[T] B. S. Tsirelson, Not every Banach space contains $\ell_{p}$ or $c_{0}$, Funct. Anal. Appl. 8 (1974), 138-141.

\begin{abstract}
We construct a Banach space that does not contain any infinite unconditional basic sequence and investigate further properties of this space. For example, it has no subspace that can be written as a topological direct sum of two infinite-dimensional spaces. This property implies that every operator on the space is a strictly singular perturbation of a multiple of the identity. In particular, it is either strictly singular or Fredholm with index zero. This implies that the space is not isomorphic to any proper subspace.
\end{abstract}

Trinity College, Cambridge, CB21TQ England

Current address: Department of Mathematics, University College London, Gower Street, London WC1E 6BT, England

E-mail address: wtg10@phx.cam.ac.uk

Université Paris ViI, U.F.R. De Mathématiques, 2 Place Jussieu, 75251 Paris Cedex 05, France

Current address: Equipe d'Analyse et Mathématiques Appliquées, Université de Marne la Vallée, 93160 Noisy le Grand, France

E-mail address: maurey@logique.jussieu.fr 